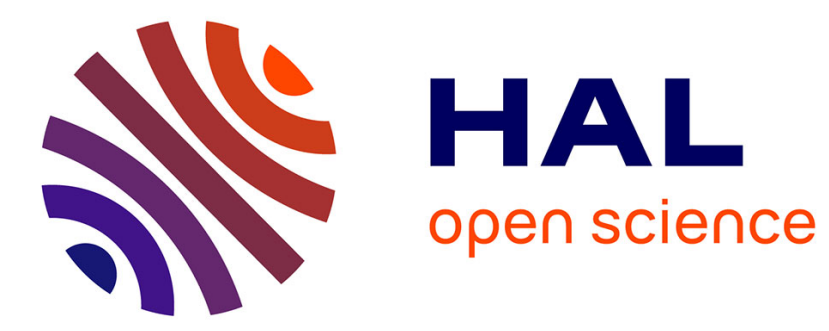

\title{
Light Scattering by Single Natural Ice Crystals
}

Valery Shcherbakov, Jean-François Gayet, Brad Baker, Paul A Lawson

\section{To cite this version:}

Valery Shcherbakov, Jean-François Gayet, Brad Baker, Paul A Lawson. Light Scattering by Single Natural Ice Crystals. Journal of the Atmospheric Sciences, 2006, 63 (5), pp.1513 - 1525. 10.1175/JAS3690.1 . hal-01823427

\section{HAL Id: hal-01823427 \\ https://hal.uca.fr/hal-01823427}

Submitted on 9 Jun 2021

HAL is a multi-disciplinary open access archive for the deposit and dissemination of scientific research documents, whether they are published or not. The documents may come from teaching and research institutions in France or abroad, or from public or private research centers.
L'archive ouverte pluridisciplinaire HAL, est destinée au dépôt et à la diffusion de documents scientifiques de niveau recherche, publiés ou non, émanant des établissements d'enseignement et de recherche français ou étrangers, des laboratoires publics ou privés. 


\title{
Light Scattering by Single Natural Ice Crystals
}

\author{
VALERY SHCHERBAKOV \\ Institute of Physics, Minsk, Belarus, and OPGC, Laboratoire de Météorologie Physique, UMR/CNRS 6016, \\ Université Blaise Pascal, Clermont-Ferrand, France \\ JEAN-FrANÇOIS GAYET \\ Laboratoire de Météorologie Physique, UMR/CNRS 6016, Université Blaise Pascal, Clermont-Ferrand, France \\ Brad BAKer ANd PAUl Lawson \\ SPEC Inc., Boulder, Colorado
}

(Manuscript received 15 March 2005, in final form 30 June 2005)

\begin{abstract}
During the South Pole Ice Crystal Experiment, angular scattering intensities (ASIs) of single ice crystals formed in natural conditions were measured for the first time with the polar nephelometer instrument. The microphysical properties of the ice crystals were simultaneously obtained with a cloud particle imager. The observations of the scattering properties of numerous ice crystals reveal high variability of the ASIs in terms of magnitude and distribution over scattering angles. To interpret observed ASI features, lookup tables were computed with a modified ray tracing code, which takes into account the optical geometry of the polar nephelometer. The numerical simulations consider a wide range of input parameters for the description of the ice crystal properties (particle orientation, aspect ratio, surface roughness, and internal inclusions). A new model of surface roughness, which assumes the Weibull statistics, was proposed. The simulations reproduce the overwhelming majority of the observed ASIs features and trace very well the quasi-specular reflection from crystal facets. The discrepancies observed between the model and the experimental data correspond to the rays, which pass through the ice crystal and are scattered toward the backward angles. This feature may be attributed to the internal structure of the ice crystals that should be considered in modeling refinements.
\end{abstract}

\section{Introduction}

Ice clouds, especially cirrus, play an important role in the energy balance of the earth-atmosphere system through their interactions with solar and terrestrial radiation (Liou 1986, 1992; Stephens et al. 1990). Their radiative properties are governed by the ice crystals' optical characteristics. The accurate modeling of cirrus single-scattering parameters is of importance in general circulation models (Kristjánsson et al. 2000). The knowledge of these parameters is the prior condition for the interpretation of remote sensing measurements of cirrus clouds (Labonnote et al. 2000). Theoretical

Corresponding author address: Dr. J.-F. Gayet, Laboratoire de Météorologie Physique, Université Blaise Pascal, 24 Avenue des Landais, 63177 Aubiére CEDEX, France.

E-mail: J.F.Gayet@opgc.univ-bpclermont.fr and numerical techniques, laboratory measurements, and practical applications of light scattering by nonspherical particles were reviewed in the book edited by Mishchenko et al. (2000). Despite the substantial progress in this domain, the optical characteristics of ice crystals measured in natural conditions are still needed, first of all for validation of numerical techniques and the determination of free parameters of models.

Jourdan et al. (2003) reported the statistical characteristics of mixed-phase and cirrus cloud-phase functions obtained from airborne measurements by the polar nephelometer (Gayet et al. 1998). The results concern datasets recorded for ensembles of cloud particles, and can be used for validation of techniques that model the phase function of randomly orientated ice crystals. At the same time, because ice particle shape and other characteristics vary greatly, it is difficult to directly compare scattering observations with theoretical simu- 
lations. Therefore, the assessment of scattering properties related to single ice crystals is of particular interest. Light scattering by single levitated ice crystals was measured by Pluchino (1986), and by Bacon and Swanson (2000). Scattering of light from atmospheric ice analogues was reported by Ulanowski et al. (2003). Lawson et al. (1998) and Barkey et al. (2002) presented phase function measurements related to ice crystals generated in a cloud chamber. However, to our knowledge, scattering properties of single natural ice crystals have not yet been measured.

The aims of this paper are (i) to present typical examples of single ice crystal angular scattering intensities (ASIs) measured for the first time in natural conditions, and (ii) to show the outcomes that were typical for the modeling of the measured ASIs. The instruments and the experimental conditions are described first. The ray tracing technique used to compute the lookup table is presented in section 3. Section 4 is devoted to the fitting of the measured ASIs on the base of the lookup table.

\section{Instrumentation, data processing, and experimental conditions}

The South Pole Ice Crystal Experiment (SPICE) was carried out at the Amundsen-Scott South Pole Station (SPS; 2835-m elevation) from 23 January to 5 February 2002. The polar nephelometer (PN) and a Stratton Park Engineering Company Inc. (SPEC) cloud particle imager (CPI; Lawson 1997) were installed on the roof of a small building at the SPS in order to simultaneously measure scattering and microphysical properties of ice crystals (see Fig. 1). The PN and the CPI were connected together using a custom-designed plumbing system with inlet funnels to maximize the probability that particles would pass through the sample volumes of both the CPI and PN. A constant flow exhaust fan was connected to the exit region of the PN.

Before the plumbing system was fabricated out of aluminum, the plumbing system and the sample tubes of the CPI and PN were constructed using Plexiglas so that flow within the system could be visualized. A fog generator was used to make a cloud that could be viewed using a laser illumination system. The flow visualization tests showed that the sample air was laminar and passed through both the CPI and PN sensitive volumes. The aluminum plumbing was fabricated and the flow rate through the system was measured using miniature pitot tubes. The velocity profiles were measured in the sample volumes of the CPI and PN. It was shown that the velocity profiles in each instrument are relatively flat and the measurements were repeatable. The uncertainty in the size-dependent acceptance of the inlet was not quantified.

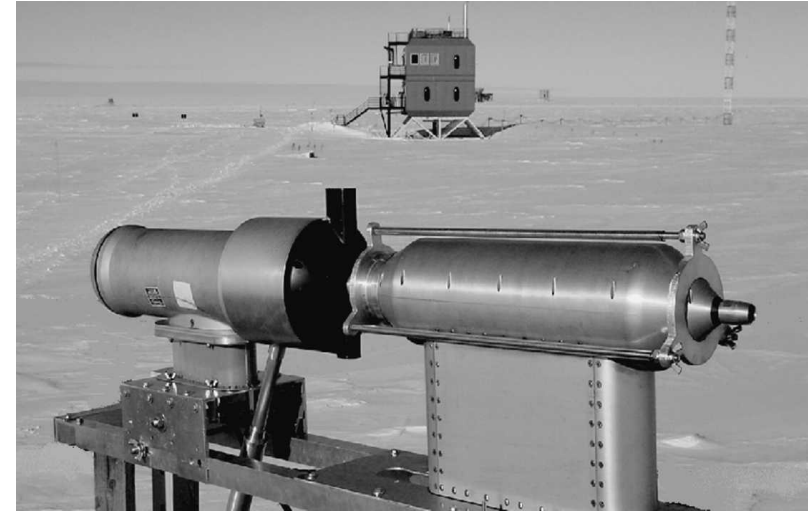

FIG. 1. CPI (right) and PN (left) installed in tandem at the Amundsen-Scott South Pole station during the SPICE experiment.

The same volume of air populated with ice crystals was passing through both instruments with a linear airspeed of about 34 and $11 \mathrm{~m} \mathrm{~s}^{-1}$ at the CPI and PN sampling volumes, respectively. Because the two instruments have different sampling areas $\left(0.5\right.$ and $0.039 \mathrm{~cm}^{2}$ for the PN and CPI instruments, respectively) that are spaced at $70 \mathrm{~cm}$, the respective measurements cannot address the same ice crystals (the probability of the coincident particle-by-particle events is low). Nevertheless, the extinction coefficients calculated from the data of both the polar nephelometer and the cloud particle imager and smoothed with a 9-s moving window were compared. The results (not shown here) highlight a very good linear correlation (0.90) between the two independent measurements. This means that the two instruments sampled statistically the same populations of ice particles.

\section{a. Polar nephelometer}

The optical scheme of the polar nephelometer (Gayet et al. 1997) is shown in Fig. 2. The ice crystals are pushed through the volume scattering chamber and intersect a collimated laser beam (operating at the wavelength of $0.8 \mu \mathrm{m}$ ) near the focal point of a paraboloidal mirror. The cross section of the laser beam has a circular shape and the intensity profile is approximately rectangular. The scattered intensities are measured at scattering angles from about $5^{\circ}$ to $169^{\circ}$ by two rows of photodiodes located in the upper and lower parts of a circular array (see Fig. 2). Therefore, the PN provides the angular scattering intensities as a two-dimensional scattering pattern defined by the scattering angle $\theta$ and by the azimuthal angle $\varphi$. The last one takes only two values that differ by $180^{\circ}$. Accordingly, the notations upward and downward angles will be used to indicate the dependence on the azimuthal angle of ASIs. 


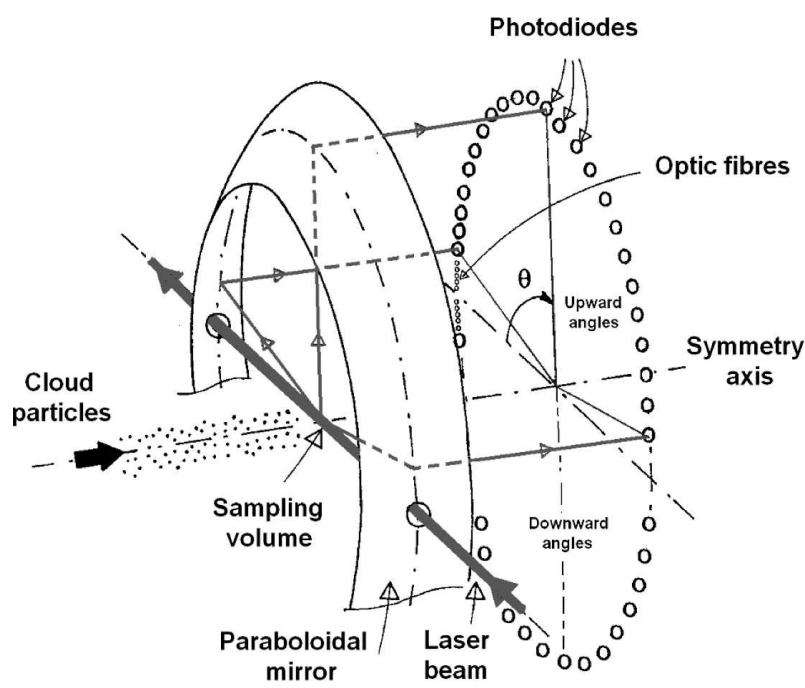

FIG. 2. Optical scheme of the polar nephelometer.

Scattering phase functions related to an ensemble of randomly oriented ice particles do not depend on the azimuthal angle. In this case, ASIs measured at the upward and downward angles are identical and the scattering properties can be described between $5^{\circ}$ and $169^{\circ}$ (see, e.g., Auriol et al. 2001). On the contrary, for single ice crystal sampling, the upward and downward ASIs have mostly different magnitude and distribution over scattering angles, and depend on the orientation of the ice crystal in the sampling volume. Consequently, the PN provides additional important information related to the two azimuthal angles $\varphi$ when single nonspherical particles are measured.

The data acquisition system of the polar nephelometer is designed to provide a continuous sampling volume by integrating the measured signals of each detector at a selected period. For instance the sampling volume $(v)$ is determined by the sampling area $(10-\mathrm{mm}$ long and 5-mm diameter beam) multiplied by the airspeed (approximately $11 \mathrm{~m} \mathrm{~s}^{-1}$ ), that is, $5.5 \mathrm{~cm}^{3}$ for the acquisition frequency of $100 \mathrm{~Hz}$. Because the concentration of ice crystals measured by the CPI during the considered time sequence was typically lower than 0.15 $\mathrm{cm}^{-3}$, single ice particles were measured. Figure 3 displays an example of time series (500 $\mathrm{m} \mathrm{s}^{-1}$ duration) of the raw intensities measured at scattering angles of $15^{\circ}$, $92^{\circ}$, and $155^{\circ}$, respectively. The results clearly evidence individual peaks, which characterize single particle events. These peaks are superimposed on background signals (or zero baselines), which are due to both electronic and optical noises. As exemplified on Fig. 3, the background signals depend on the scattering angles and are ranged from about 5\% (of the signal dynamical range) for the smallest forward and largest backward

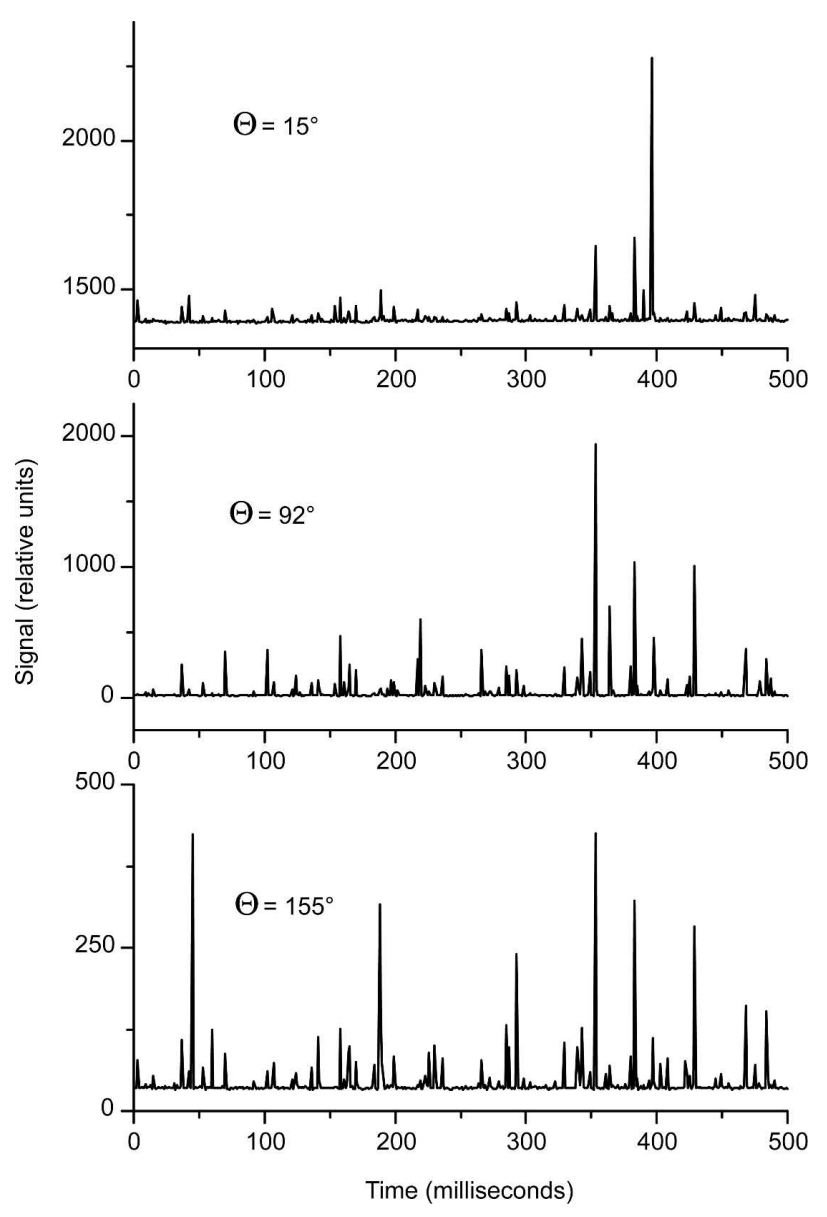

FIG. 3. Example of time series of the raw scattering intensities measured at scattering angles of $15^{\circ}, 92^{\circ}$, and $155^{\circ}$.

angles to $0.5 \%$ for the sideward angles. Also, there are low frequency variations (not shown) of the background signals. Those variations are substantial for measurements with a low signal-to-noise ratio. The wavelet denoising technique (see, e.g., Rao and Bopardikar 1998) was employed to compute time series of the background signals at each channel. The accuracy of the ASIs measurements was significantly improved by removing the time-dependent background signals. In addition, a special routine was used in order to check whether the registered signals from a particle are within the linear range of the detectors. The ASIs of that particle were rejected if a signal at any angle did not satisfy that condition.

Table 1 summarizes the scattering angles and the accuracies of the measured intensities. The accuracies were estimated by considering the variations in results of calibration that was performed several times, the noise of the photodiodes, the errors of the digitizing, the variations of the particle position within the sensitive volume, and the laser beam divergence that caused 
TABLE 1. Channel numbers and corresponding scattering angles measured by the polar nephelometer. The dots indicate the available channels during the SPICE experiment. The accuracies on the measured scattering intensities are also reported. The left part and right parts of the table relate the upward and downward scattering angles, respectively (see text for details).

\begin{tabular}{|c|c|c|c|c|c|c|c|}
\hline \multicolumn{4}{|c|}{ Upward angles } & \multicolumn{4}{|c|}{ Downward angles } \\
\hline $\begin{array}{l}\text { Channel } \\
\text { number }\end{array}$ & $\begin{array}{l}\text { Scattering } \\
\text { angle }\left({ }^{\circ}\right)\end{array}$ & OK & Accuracy & $\begin{array}{l}\text { Channel } \\
\text { number }\end{array}$ & $\begin{array}{l}\text { Scattering } \\
\text { angle }\left({ }^{\circ}\right)\end{array}$ & OK & Accuracy \\
\hline 01 & 4.28 & & & 56 & 3.49 & & \\
\hline 02 & 5.86 & $\bullet$ & $50 \%$ & 55 & 5.07 & & \\
\hline 03 & 7.44 & $\bullet$ & $50 \%$ & 54 & 6.65 & & \\
\hline 04 & 9.02 & $\bullet$ & $50 \%$ & 53 & 8.23 & & \\
\hline 05 & 10.6 & $\bullet$ & $50 \%$ & 52 & 10.6 & & \\
\hline 06 & 15.0 & $\bullet$ & $5 \%$ & 51 & 18.5 & $\bullet$ & $5 \%$ \\
\hline 07 & 22.0 & $\bullet$ & $5 \%$ & 50 & 25.5 & $\bullet$ & $3 \%$ \\
\hline 08 & 29.0 & $\bullet$ & $3 \%$ & 49 & 32.5 & & \\
\hline 09 & 36.0 & $\bullet$ & $3 \%$ & 48 & 39.5 & $\bullet$ & $3 \%$ \\
\hline 10 & 43.0 & $\bullet$ & $3 \%$ & 47 & 46.5 & $\bullet$ & $3 \%$ \\
\hline 11 & 50.0 & $\bullet$ & $3 \%$ & 46 & 53.5 & $\bullet$ & $3 \%$ \\
\hline 12 & 57.0 & $\bullet$ & $3 \%$ & 45 & 60.5 & & \\
\hline 13 & 64.0 & $\bullet$ & $3 \%$ & 44 & 67.5 & & \\
\hline 14 & 71.0 & $\bullet$ & $3 \%$ & 43 & 74.5 & & \\
\hline 15 & 78.0 & $\bullet$ & $3 \%$ & 42 & 81.5 & & \\
\hline 16 & 85.0 & $\bullet$ & $3 \%$ & 41 & 88.5 & & \\
\hline 17 & 92.0 & $\bullet$ & $3 \%$ & 40 & 95.5 & & \\
\hline 18 & 99.0 & • & $3 \%$ & 39 & 102.5 & & \\
\hline 19 & 106.0 & - & $3 \%$ & 38 & 109.5 & $\bullet$ & $3 \%$ \\
\hline 20 & 113.0 & • & $3 \%$ & 37 & 116.5 & & \\
\hline 21 & 120.0 & $\bullet$ & $3 \%$ & 36 & 123.5 & & \\
\hline 22 & 127.0 & • & $3 \%$ & 35 & 130.5 & & \\
\hline 23 & 134.0 & & & 34 & 137.5 & • & $5 \%$ \\
\hline 24 & 141.0 & $\bullet$ & $5 \%$ & 33 & 144.5 & $\bullet$ & $10 \%$ \\
\hline 25 & 148.0 & $\bullet$ & $10 \%$ & 32 & 151.5 & $\bullet$ & $15 \%$ \\
\hline 26 & 155.0 & $\bullet$ & $20 \%$ & 31 & 158.5 & & \\
\hline 27 & 162.0 & $\bullet$ & $30 \%$ & 30 & 165.5 & & \\
\hline 28 & 169.0 & $\bullet$ & $50 \%$ & 29 & 172.5 & & \\
\hline
\end{tabular}

the much higher measurement errors at the small forward and large backward angles. The optical aperture of the receivers at the angles $<15^{\circ}$ is smaller than that one at other angles, which increases the sensitivity of the measurements to the particle position and, therefore, errors at the small angle range. Concerning the possible effect on results of particle spinning, it should be noticed the following. Passing through the sensitive volume within the time period of $\sim<0.45 \mathrm{~m} \mathrm{~s}^{-1}$, a particle has to revolve at the angular speed of $12 \mathrm{rev} \mathrm{s}^{-1}$ so that the rotation deflects the scattered light at the angle equal to the field-of-view of the detectors (i.e., $2^{\circ}$ ). Since the flow through the sample tubes was laminar, the spinning of particles was much lower than $12 \mathrm{rev}$ $\mathrm{s}^{-1}$, and the subsequent errors can be neglected.

During SPICE experiment, there were available 26 upward and 9 downward channels (see Table 1). The measurements were limited to nonpolarized light. Because of the calibration procedure (Gayet et al. 1997), the absolute values of ASIs were measured. That provides possibility to estimate size of a particle. The PN integrated signals during an acquisition period $(0.01 \mathrm{~s})$, which included the time interval $\left(\sim<0.45 \mathrm{~m} \mathrm{~s}^{-1}\right)$ when a particle was within the sensitive volume. The size of the particles was estimated with the assumption that they passed the sensitive volume along the diameter of the cross section. The value of the size, listed in Table 3 , should be considered as the lower bound. If the particle passed outside the diameter, the crystal should be larger to give the same scattered energy being $n$ the sensitive volume shorter period of time.

\section{b. Cloud particle imager}

The CPI casts an image of a particle on a solid-state, one-million-pixel digital charge-coupled device (CCD) camera by freezing the motion of the particle using a 40-ns pulsed, high-power laser diode (Lawson et al. 2001). A particle detection system (PDS) with upstream lasers precisely defines the focal plane so that at least one particle in the image is almost always in focus. Each pixel in the CCD camera array has an equivalent 
size in the sample area of $2.3 \mu \mathrm{m}$, so particles of sizes from approximately $10 \mu \mathrm{m}$ to $2 \mathrm{~mm}$ are imaged. The shadow depth of each pixel can be expressed in up to 256 gray levels and the refresh rate of the CCD camera is $40 \mathrm{~Hz}$. A video-processing engine identifies and sizes particles within the one-million-pixel array, saving only the regions of interest.

The sample area of the PDS $(0.707 \times 2.35 \mathrm{~mm} \times 2.35$ $\mathrm{mm})$ times the flow rate of $34 \mathrm{~m} \mathrm{~s}^{-1}$ and results in a CPI sample rate of $0.13 \mathrm{~L} \mathrm{~s}^{-1}$, and this is the sample rate used to compute particle concentrations presented in this work. However, ice particles have inertia and are affected by gravity differently than the air. That is, particles do not completely follow the airflow. This results in large uncertainties in the measurement of the PDS, total concentration, extinction, and mass content. It was observed, by varying the orientation of a CPI, that the measurements were sensitive to the instruments' orientation relative to the wind. Pointing a CPI into the wind, which is how nearly all the data was collected, seemed to maximize the number of small particles observed. Pointing vertically maximized the number of large precipitation particles observed. Since there was no way to make quantitative measurements of the uncertainty in particle flow rate, we cannot quote uncertainties in ice crystal concentration measurements. Furthermore, the probability of triggering the CPI's PDS increases with particle size. However, given the large uncertainties just discussed and because the PN must also have a roll off with decreasing size as well, for the data presented herein, no adjustments were made to the data to account for triggering probabilities. It is noteworthy that while the absolute quantitative measurements have large and unspecific uncertainties, the CPI and PN must have sampled quite similar crystal populations since they were connected in tandem on the same sample tube. Theoretically it is expected that the PN would have some bias toward the smaller of the crystals sampled by the CPI due to gravitational settling during transit from the CPI sample volume to the PN sample volume. Again, this bias is undetermined. The CPI were generally operated for approximately 12 to 18 $\mathrm{h}$ day $^{-1}$, unless an instrument was shut down because of electrical outages or other interruptions at SPS.

\section{c. Experimental conditions}

During the SPICE experiment, several episodes of diamond dust (or clear sky precipitation, see, e.g., Kikuchi and Hogan 1979) were documented. The data that are discussed in this paper were obtained on 25 January 2002. During the data-sampling time period (from 2256:00 to 2312:30 UTC), the pressure was 676.9 $\mathrm{hPa}$, the surface air temperature and the dewpoint
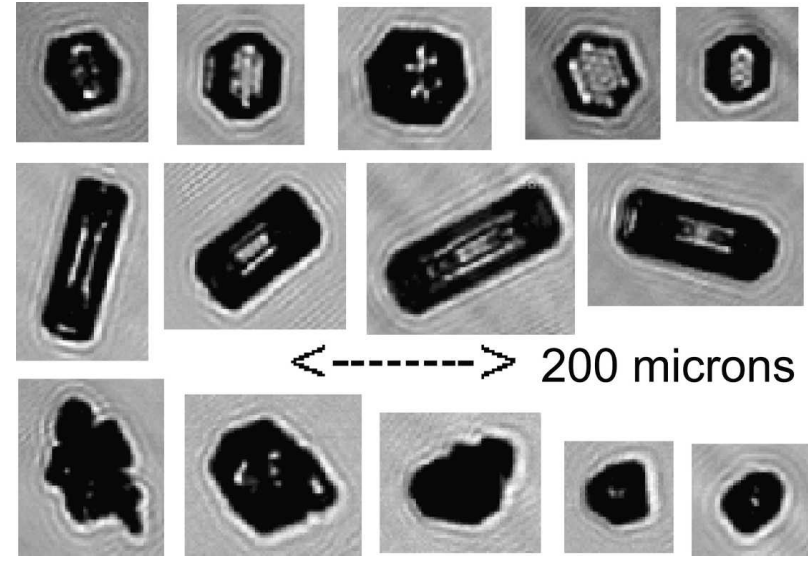

FIG. 4. Typical images of ice particles recorded by the CPI instrument.

temperature were $-30.0^{\circ}$ and $-32.2^{\circ} \mathrm{C}$, respectively. We note in passing that the meteorological parameters are provided by the National Oceanic and Atmospheric Administration (NOAA) Climate Monitoring and Diagnostics Laboratory, Boulder, Colorado (Herbert et al. 1986). Diamond dust ice particles and optical-related $22^{\circ}$ halo feature were observed during this period of measurements. Typical particle images sampled by the CPI are shown in Fig. 4. Most particles were hexagonal ice columns or plates. Table 2 shows the percentage of ice crystal habits in terms of their number concentration, area, and mass for the considered sampling period. Results in Table 2 show that in terms of the area percentage contribution of crystal habits $48.5 \%$ was hexagonal plates, columns, or thick hexagonal plates/ columns, while about $18.8 \%$ was classified as irregular particles or complex crystals with side planes. If small plates and spheroids were not taken into account, the mean particle size was about $85 \mu \mathrm{m}$ during the considered time sequence.

\section{The Monte Carlo ray tracing model}

A Monte Carlo ray tracing method devised by Macke et al. (1996a,b) was used to model the measured

TABLE 2. Percentage contributions of crystal habits to the number concentration, area, and mass. The measurements are based on the CPI samples obtained on 25 Jan 2002 between 2256:00 and 2312:30 UTC. [Lco (long columns), Plt (plates), Sco (short columns and thick plates), Csp (complex crystals with side planes), Irr (irregular), Shx (small plates and spheroids).]

\begin{tabular}{lrrrrrr}
\hline Type & Lco & Plt & Sco & Csp & \multicolumn{1}{c}{ Irr } & Shx \\
\hline Number & 23.5 & 6.4 & 10.4 & 0.5 & 9.6 & 49.6 \\
Area & 32.5 & 5.4 & 10.6 & 2.0 & 16.8 & 32.7 \\
Mass & 24.9 & 6.2 & 9.5 & 2.9 & 21.6 & 34.9 \\
\hline
\end{tabular}


angular scattering intensities. Some modifications were made in order to adapt the code to the PN optical scheme (including the optical aperture of the receivers), that is, to provide the computation of $2 \mathrm{D}$ scattering patterns of a single ice crystal having a specified orientation in the sampling volume. The modifications do not affect the physical grounds of the code.

The geometry of light scattering by a hexagonal particle is shown in Fig. 5. The incident light direction is opposite to the axis $Z$. The scattering angle $\theta$ is defined as the angle between the scattering and incident-beam directions. The azimuthal angle $\varphi$ is the angle between the axis $X$ and the projection of the scattering direction onto the $X-Y$ plane. The upward and downward ASIs correspond to $\varphi=0^{\circ}$ and $\varphi=180^{\circ}$, respectively. The particle orientation is defined by the Euler angles $\alpha, \beta$, and $\gamma$. The case with zero values of all Euler angles is shown in Fig. 5. The angle $\alpha$ corresponds to the righthand rotation of the particle about the axis $Z$, the angle $\beta$ defines the right-hand rotation about the new axis $X^{\prime}$, and the angle $\gamma$ corresponds to the right-hand rotation about the new axis $Z^{\prime}$.

An approximation of small-scale surface roughness based on tilted angle distribution is included in the Macke et al. (1996a,b) code. Each time a ray hits a crystal surface, the normal to this surface is tilted with respect to its original direction by a certain angle. The reflection and refraction directions are computed with respect to this tilted surface element. The tilt angle is defined by random numbers, which are uniformly distributed between zero and a given maximum value. It was found that measured ASIs are poorly modeled when the tilt angles are uniformly distributed within some interval. This was especially the case for the angular scattering intensities corresponding to the quasispecular reflection from one of crystal facets (see section 4). Consequently, we included in the code a new model of the azimuthally homogeneous roughness (see details in appendix). The model is based on the Weibull statistics (see, e.g., Dodson 1994). The tilt-angles probability distribution function (PDF) is described by the scale $\sigma$ and shape $\eta$ parameters. The approach incorporates the Cox and Munk (1954) model (see also Yang and Liou 1998) when $\eta$ takes the value of 1.0.

Air bubbles may be trapped inside rapidly growing ice particles (Macke et al. 1996b; Labonnote et al. 2000). The Macke et al. code allows simulations of light scattering by internal inclusions. For the air bubbles model, we used the microphysical and optical parameters proposed by Macke et al. (1996b). The mean free pathlength (MFPL) between two subsequent inclusions can be varied.

The modified Monte Carlo ray tracing code (hereafter the modified code) was thoroughly tested. For in-

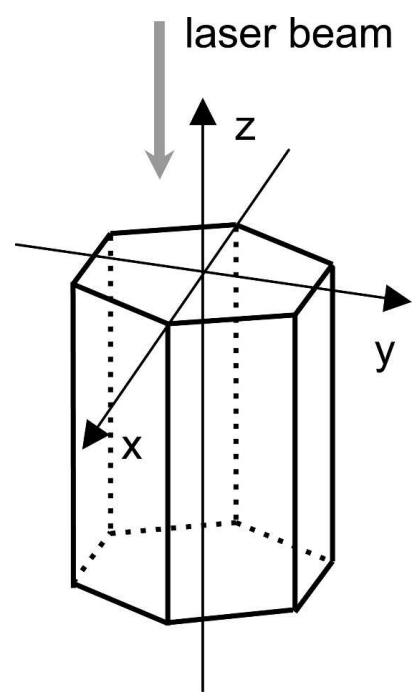

FIG. 5. Geometry of light scattering by a hexagonal particle.

stance, it reproduces very well all peaks show by the trace 3 in Fig. 2 of Ulanowski et al. (2003) with, of course, identical input parameters. The peaks vanish if the particle is rotated with $\Delta \beta$ higher than the half of the field of view of the receivers. On the other hand, the peaks are decreasing and broadening when $\sigma$ is increasing.

A lookup table of the single ice crystal ASIs was generated using the modified code. The crystal is assumed to be a hexagonal column/plate. The input parameters are the three Euler angles, the aspect ratio of the ice crystal, the two parameters of the surface roughness, and the MFPL, respectively. The ASIs were calculated assuming: (i) the incident wavelength of $0.8 \mu \mathrm{m}$, (ii) the complex refractive index for ice tabulated by Warren (1984), and (iii) the ice crystal minimum dimension of about $20 \mu \mathrm{m}$. It should be noticed that the normalized ASIs are essentially not particle-sizedependent in the range of PN documented scattering angles. Each angular scattering intensity was computed with the number of incident rays of $10^{6}$. Considering the number of the input parameters and corresponding grids, the lookup table generation is extremely time consuming. It was a little reduced by considering the symmetry of a hexagonal particle. The lookup table was used to fit the measured ASIs (at all 35 angles simultaneously) and then to retrieve the corresponding parameters of single ice crystals.

\section{Interpretation of the scattering properties of single natural ice crystals}

\section{a. Shortcomings of the lookup table interpretation}

It should be stressed that an assessment of seven characteristics of a particle (the Euler angles, the aspect 
ratio, the surface roughness parameters, and the MFPL) on the base of the lookup table inputs from the measured ASIs is an extremely challenging task. This is due to not only the high number of the dimensions of the lookup table, but also to the essential nonlinearity of the relationship between the particle parameters and the ASIs. Therefore, when a maximum-likelihood method is used to solve the inverse problem, numerous solutions could be appropriate if errors of measurements are considered. For example, if the axis of a hexagonal column is perpendicular to the incident light $\left(\beta=90^{\circ}\right)$, the normalized ASIs are insensitive to the aspect ratio. Another example is that smooth featureless ASIs can be usually matched by a large set of different input parameters.

Consequently, retrievals were mostly performed for ASIs having distinguishing features characterized by a local maximum (or maxima) related to quasi-specular reflections. Because of the nonlinearity mentioned above, interpolation of the lookup table is not applied for the particle parameters retrievals. Instead, the parameters values are found for each case on the grid of the lookup table, and a set of intermediate solutions is considered according to the level of measurement errors. Around those solutions, the grid of the lookup table is refined, and the final solution is obtained from this refined grid. Of course, the described algorithm is time consuming, and it often requires operator supervision. However, such an approach assures more rigorous treatment of the nonlinearity. In addition, it should be noted that the logarithms of the ASIs are fitted and a weight matrix is used for considering the lower accuracy of the measurements in the range of scattering angles between 5 and 15 degrees compared to other angles (see Table 1). Also, there were fitted only those ASIs that belong to time periods where each occurrence of the signal from an ice crystal in the PN time series was surrounded by several strobes of the background signal. That is, the probability of fitting ASIs that were due to two particles was very low.

\section{b. Interpretation of the results}

The overwhelming majority of the recorded ASIs reveal strong distinguishing features. A representative example of such angular scattering intensities measured at 2300:16.52 UTC is shown in Fig. 6a. The upward and downward measurements are plotted with full circles and open triangles, respectively. Results of Fig. 6a highlight a very well-marked upward peak centered at $\theta=$ $68^{\circ}$ with a magnitude even higher than the scattering intensity at $5.8^{\circ}$. [The broadness of peaks was also observed by Ulanowski et al. (2003) during the hexagonal glass fiber experiment.] A smaller local upward maxi- mum is also observed at $141^{\circ}$. Concerning the downward feature, the scattering angles are less documented: 9 angles against 26 for the upward side (see Table 1). However, Fig. 6a shows that a peak value at $109.5^{\circ}$ is about 30 times higher than the neighbor values.

Figure 6a also superimposes the model results (black and gray curves for upward and downward angles, respectively) of the observed data. The agreement is good enough to reproduce most of the distinguishing features of the observed ASIs for both upward and downward angles between $20^{\circ}$ to $125^{\circ}$. The most important achievement is that the model simulates very well the quasi-specular reflection. We note in passing that the term quasi-specular is used to describe a reflection from a rough surface, which differs from the perfect plane specular reflection, that is, the deltalike peak of the intensity. The better agreement is obtained when the roughness parameter $\eta$ takes the value of 0.75 ; but it becomes not so good when $\eta$ is restricted to 1.0 (the Cox-Munk roughness model). From the analysis of the 2D scattering patterns of the corresponding pristine crystal, the $68^{\circ}$ and $109^{\circ}$ local maxima are due to the quasi-specular reflection from the side and the hexagonal facets, respectively. According to the orientation of the ice crystal (see Euler angles retrieved values below), the peaks of the reflections were directed alongside the receiving photodiodes. Therefore, the measured ASIs correspond to the wings of the pattern of the quasi-specular reflection. Figure 6a reveals significant differences between the observed and model ASIs for scattering angles ranged from $125^{\circ}$ to $160^{\circ}$. These differences are much larger than the measurement error estimates and cannot be attributed to instrument shortcomings. As a matter of fact, the featureless ASIs were well reproduced when nonsymmetric ice crystals were sampled (Baran et al. 2005). In addition, the scattering phase functions of water clouds, measured by the PN during other field campaigns, were found in very good agreement with the corresponding microphysical data and the Mie theory (Gayet et al. 2002). Consequently, the discrepancies at backward angles may be attributed to the model, which probably does not consider unexpected optical interactions within the structure of the ice crystals.

The retrieved parameters of the ice crystal are listed in Table 3 (case A). We note in passing that the error range in the estimates shown in Table 3 corresponds to a projection of the measurement errors into the space of the assessed parameters. The three Euler angles are $268^{\circ}, 34^{\circ}$, and $34^{\circ}$, the aspect ratio is 5 (elongated column), the roughness parameters $\sigma$ and $\eta$ are 0.75 and 0.1 , respectively. The estimated size parameter $(D=71$ $\mu \mathrm{m})$ of the ice crystal is also reported in Table 3. 

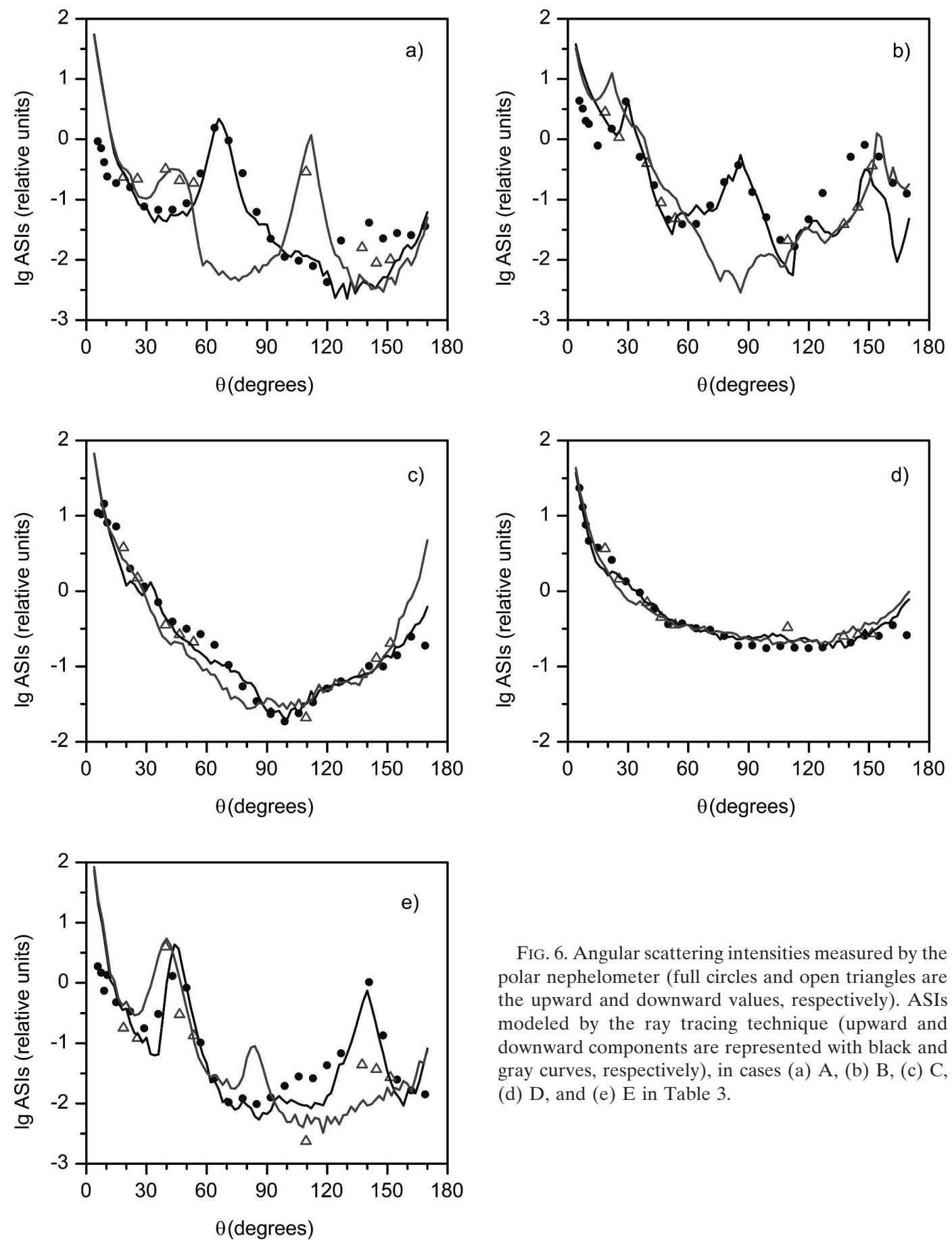

FIG. 6. Angular scattering intensities measured by the polar nephelometer (full circles and open triangles are the upward and downward values, respectively). ASIs modeled by the ray tracing technique (upward and downward components are represented with black and gray curves, respectively), in cases (a) A, (b) B, (c) C, (d) D, and (e) E in Table 3.

Here, $D$ is the diameter of the circle circumscribing the hexagonal facet of the ice crystal. Table 3 reports the MFPL ratio, which is equal to the mean free pathlength divided by the parameter $D$. The ratio is quite high. It means that a scattering pattern of such a kind corresponds to particles having a small amount of internal inclusions. And, the effect of air bubbles on the ASIs is rather small in this particular case. As explained in section 2, a single ice crystal sampled by the PN cannot be exactly identified within the CPI images (and vice versa). Nevertheless, very close to the PN sampling time, the measured CPI images reveal that hexagonal ice columns or plates are the most probable coincident ice particles. 
TABLE 3. Retrieved values of the ice crystal parameters related to four examples of measurements: the three Euler angles $\alpha$, $\beta$, and $\gamma$, which define the particle orientation; the aspect ratio; the surface roughness parameters $\eta$ and $\sigma$; the MFPL ratio; and the diameter $D$ of the circle circumscribing the hexagonal facet an ice crystal.

\begin{tabular}{lcccrr}
\hline \hline Parameter & Case A & Case B & Case C & Case D & Case E \\
\hline$\alpha^{\circ}$ & $268 \pm 2$ & $181 \pm 2$ & $192 \pm 2$ & $44 \pm 5$ & $266 \pm 2$ \\
$\beta^{\circ}$ & $34 \pm 2$ & $79 \pm 2$ & $32 \pm 2$ & $64 \pm 5$ & $70 \pm 2$ \\
$\gamma^{\circ}$ & $34 \pm 2$ & $15 \pm 2$ & $20 \pm 2$ & $40 \pm 5$ & $32 \pm 2$ \\
Aspect ratio & $5 \pm 1$ & $2.5 \pm 0.25$ & $1 \pm 0.25$ & $5 \pm 1$ & $0.5 \pm 0.25$ \\
$\eta$ & $0.75 \pm 0.02$ & $0.75 \pm 0.02$ & $0.75 \pm 0.02$ & $0.75 \pm 0.02$ & $0.75 \pm 0.02$ \\
$\sigma$ & $0.1 \pm 0.02$ & $0.15 \pm 0.02$ & $0.25 \pm 0.02$ & $0.25 \pm 0.05$ & $0.1 \pm 0.02$ \\
MFPL ratio & $10 \pm 1$ & $8 \pm 1$ & $8 \pm 1$ & $1 \pm 0.5$ & $10 \pm 1$ \\
$D, \mu$ m & 71 & 27 & 44 & 26 & 105 \\
\hline
\end{tabular}

Another representative example is shown in Fig. 6b with the same symbols as in Fig. 6a. The ice crystal was sampled at 2257:02.47 UTC. The results highlight three local maxima of the upward ASIs at the scattering angles of $29^{\circ}, 85^{\circ}$, and $148^{\circ}$. From the analysis of the 2D scattering patterns of the corresponding crystal, the first maximum $\left(29^{\circ}\right)$ corresponds to the refracted rays passed through the crystal, the second one $\left(85^{\circ}\right)$ is the wing of the quasi-specular external reflection, and the third $\left(148^{\circ}\right)$ is due to internal reflections. The agreement between the model and the experimental data is quite well. The first two maxima of the upward ASIs are very well fitted. The model predicts the maximum at $148^{\circ}$ but with the narrower angular distribution and the lower magnitude comparing to the PN observation. As in the previous example, discrepancies are evidenced at the backward angles. The retrieved parameters are listed in Table 3 (case B). The aspect ratio is 2.5 (elongated column) and the size parameter is evaluated to $27 \mu \mathrm{m}$.

Despite that smoothed ASIs features represent only a few percent of the overall optical properties of the considered samples, we display on Figs. 6c,d two representative examples of smoothed ASIs with close upward and downward values. The data were sampled at 2257:56.86 and 2256:44.66 UTC, respectively. Comparing the results on the two igures, the range of the ASIs values in Fig. $6 \mathrm{c}$ is much higher than in Fig. $6 \mathrm{~d}$. The retrieved ice crystal parameters are reported in Table 3 (cases $\mathrm{C}$ and $\mathrm{D}$, respectively). Numerical simulations related to the case $\mathrm{C}$ (Fig. 6c) clearly showed that the model ASIs are sensitive to rotation of the particle. Furthermore, the assessed value of the MFPL ratio (see Table 3) is close to those ones of the cases A and B. In other words, the scattering pattern is little affected by internal inclusions. The minimum of the ASIs becomes less pronounced with decreasing of the MFPL value. The aspect ratio of the particle is found to be unity with the size parameter evaluated to $44 \mu \mathrm{m}$. All the above means that the case $\mathrm{C}$ may be a hexagonal ice crystal, which was so orientated that straight rays, refracted and reflected, went past the receiving photodiodes.

As for the case D (Fig. 6d), the retrieved results and the model ASIs are much less sensitive to the Euler angles. The MFPL ratio is considerably lower than in the above-discussed cases. The corresponding amount of internal inclusions along with the high value of the roughness parameter $\sigma$ lead to smoothed featureless scattering patterns, which have no clearly pronounced peaks. Furthermore, the ASIs have the properties similar to those ones of phase functions observed for polycrystals and irregular ice particles (see, e.g., Baran et al. 2005). Considering that, one may suggest that the case $\mathrm{D}$ is a nonsymmetric irregularly shaped ice crystal. The case D supports the hypothesis that the sensitivity to the particle shape of the scattering pattern of a large ice crystal decreases with increasing of the surface roughness and amount of internal inclusions.

Figure 6e shows an example when the 2D scattering patterns correspond to a hexagonal plate. The estimated value of the aspect ratio is of $0.5 \pm 0.25$ (see Table 3, case E). The ice crystal was sampled at 2304:29.34 UTC. The local maxima at $140^{\circ}$ upward and at $40^{\circ}$ downward angles are due to wings of the quasispecular external reflections from the side and hexagonal facets, respectively. Other maxima are formed by rays having internal reflections. The discrepancy between the measured and model ASIs in the downward angle range from $110^{\circ}$ to $150^{\circ}$ could be due to a crystal deviation from the ideal hexagonal shape. This suggestion follows from the fact that there is a noticeable local maximum at the $136^{\circ}$ downward angle when the Euler angle $\gamma$ is of $28^{\circ}$, that is, $4^{\circ}$ away from the estimated value.

\section{Discussion}

The methodology described above was used to investigate the variability of microphysical properties of ice particles. A thousand of the recorded ASIs were pro- 
cessed on the standard grid of the lookup table and about one hundred of the ASIs were modeled with the grid refinement and the operator supervision. The retrieved particles characteristics were found to vary in the following ranges. The roughness parameter $\eta$ takes values between 0.73 and 0.77 . These values indicate that the probability density function of the tilt angle is sharper than it follows from the Cox-Munk model. The parameter $\sigma$ is within the range of $0.05-0.25$, which corresponds to a deeply rough surface according to the classification of Yang and Liou (1998). The aspect ratio takes values from 0.3 to 5 indicating a wide range of geometrical properties of plates and elongated columns as observed from the CPI images (see Fig. 4). The MFPL ratio is of 8 or higher. The best fitting of the measured ASIs was occasionally achieved without considering the internal inclusions effect.

The model, as a rule, follows quite well the main features of the measured ASIs. Especially as shown in Figs. 6a,b, the maxima of the quasi-specular reflection are usually very well fitted. At the same time, we have not succeeded to achieve the flawless agreement. The discrepancies between the model and the experimental data correspond to the rays, which pass through the crystal and are then scattered toward the backward directions. These discrepancies are larger than the measurement errors, and they may be explained from hypothesized optical phenomena, which are responsible for rays spreading in the interior of the ice crystal.

Among these hypotheses we can mention soluble absorbing materials that might affect optical properties of ice. Accordingly, the imaginary part of the refractive index might vary within a large range. Internal ice structures, which form hollow columns, for instance, can be clearly evidenced from CPI images (see Fig. 4). Several types of random number generators can be proposed as a mathematical model of the probability distribution function of the subsequent tilt angles. There is no information about the effective size of internal inclusions and related optical properties. Consequently, the following characteristics and parameters of the model can be revised: (i) the value of the imaginary part of the ice refractive index, (ii) the crystal shape (hollow or pyramidal particles, bullets), (iii) statistics of surface roughness, and (iv) the effective size of air bubbles.

Effects of all above listed items and all their combinations were investigated. For example, the depth of the cavities and the height of the pyramidal tops varied over a wide range, and the asymmetry parameter of the air bubbles phase function took values from 0.5 to 0.9 . It turns out that the listed modifications do not noticeably improve the agreement between the model and the observations at scattering angles ranged between 125 and 160 degrees without a significant degradation in the fitting of the quasi-specular reflection. In our opinion, the discrepancies are due to other physical properties of crystals. For instance, it can be frequently observed from CPI images that particles are not homogeneous. There are internal structures, which look like variations of the density. The subsequent variations of the refractive index may significantly affect directions of propagation of rays passing through the crystal. Besides, the angles between the crystal side facets could differ from those ones of an ideal hexagonal particle and the diffraction on crystal facets could deflects ray traces (Hesse and Ulanowski 2003). This should be a subject of future investigations.

On the other hand, the ray tracing approach is an approximation that follows from the exact theory. Generally, an approximation should have deviations. Even when the particle surface is very smooth and has only a few elements, one needs very large size parameters in order to reduce considerably the geometrical optics errors (Mishchenko and Macke 1999). It would be better to model ASIs using an algorithm strictly based on the electromagnetic theory and, in particular, include explicitly the effects of diffraction on individual surface elements (microfacets), which tend to spread the scattered light around the specular reflection or the refraction direction (Mishchenko et al. 1997). But, there is no one single currently available method that can compute the single-scattering properties of ice crystals over the large size range (see, e.g., Baran 2004). And, in our opinion, the ray tracing approach is the best model that is available and provides possibility to compute scattering characteristics of ice particles having high values of the size parameter.

The above study discusses single particle scattering properties, and in turn it is interesting to assess the contribution of all the single events documented during the considered sample. Figure 7 displays the mean ASI (black line) obtained by averaging the measurements recorded during the 16-min time interval (7833 ice particles). Despite a very high scattering angle variability of the distinguishing ASIs features of single events (see examples in Fig. 6), it is quite satisfying to observe on Fig. 7 a rather well-known scattering phase function because the sample corresponds to an ensemble of randomly oriented particles of different habits, sizes, and aspect ratios. The $22^{\circ}$ halo peak is well marked, and is the feature that is confirmed by the observations made during the experiment. On the contrary, the $46^{\circ}$ halo peak is almost totally smoothed, which is consistent with the experimental conditions too. The model ASIs are also shown in Fig. 7. They correspond to an ensemble of randomly orientated hexagonal crystals hav- 


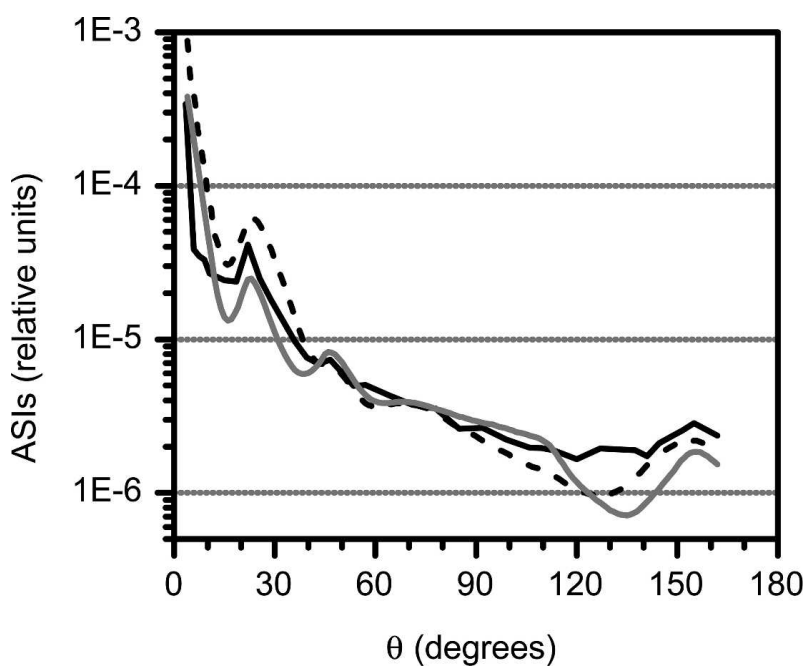

FIG. 7. Mean ASIs (black curve) and ASIs of ensembles of randomly orientated hexagonal crystals having the aspect ratio values of 0.5 (dashed curve) and 5.0 (gray curve).

ing rough surface with the parameters $\eta=0.75$ and $\sigma=$ 0.1 . Two aspect ratio values of 0.5 (dashed curve) and 5.0 (gray curve) are considered. The three curves are normalized at $\theta=43^{\circ}$. The results show that the main observed features are well modeled for scattering angles smaller than about $120^{\circ}$. The observed minimum around $130^{\circ}$ and the "ice bow" around $155^{\circ}$ are much less pronounced in comparison with the model results. Barkey et al. (2002) also reported discrepancies between observations and modeling for backward angles during laboratory experiment related to columnar shaped ice crystals. This may well be attributed to the variations of the properties of the sampled ice crystals. For instance, about $18.8 \%$ was classified as irregular particles or complex crystals with side planes (see Table 2). On the other hand, this might be the outcome of the discrepancy that was discussed in section $4 \mathrm{~b}$.

\section{Conclusions}

During the South Pole Ice Crystal Experiment (SPICE) carried out at the Amundsen-Scott South Pole Station in the austral summer of 2002, the polar nephelometer and the cloud particle imager (CPI) were deployed in order to simultaneously measure scattering and microphysical properties of natural ice crystals. Diamond dust ice particles and the optical-related $22^{\circ}$ halo feature were observed during the period of measurements considered in the paper.

For the first time, angular scattering intensities (ASIs) of single ice crystals formed in natural conditions were measured. These intensities exhibit ex- tremely high variability in magnitude and distribution over scattering angles. The very well-marked peaks are undoubtedly attributed to quasi-specular reflections from crystal facets and the refracted rays passed through the ice crystals.

A lookup table of ASIs was computed using a ray tracing code adapted to the PN optical characteristics. The input parameters of the lookup table represent most of the crystal properties, including internal inclusions, and a new model of the surface roughness, which assumes the Weibull statistics. The model ASIs reproduce the overwhelming majority of the distinguishing features of the observed measurements and therefore trace very well the quasi-specular reflections. The retrieved properties of the considered ice particle sample show that the roughness parameter $\eta$ takes values between 0.73 and 0.77 . These values indicate that the probability density function of the tilt angle is sharper than it follows from the Cox-Munk model. The parameter $\sigma$ is within the range of $0.05-0.25$, which corresponds to a deeply rough surface, and the aspect ratio takes values from 0.3 to 5 indicating a wide range of geometrical properties of plates and elongated columns. The mean free pathlength ratio is of 8 or higher.

Discrepancies between the model and the experimental data are pointed out. They correspond to the rays, which pass through a crystal and are scattered into the backward directions. These differences are much larger than the measurement error estimates and may not be attributed to instrument shortcomings. Consequently, the discrepancies at backward angles may be attributed to the model, which probably does not consider unexpected optical interactions within the structure of ice crystals. This should be a subject for future investigations.

Despite the very high scattering angle variability of the ASIs features of single events, the mean scattering phase function related to the overall contribution of single events (7833 ice particles) exhibits the wellknown scattering feature because the sample correspond to an ensemble of randomly oriented particles of different habits, sizes and aspect ratios. The $22^{\circ}$ halo peak is well marked and is confirmed by the observations made during the experiment. The main observed features are well modeled for scattering angles smaller than about $120^{\circ}$. The observed minimum around $130^{\circ}$ and the ice bow around $155^{\circ}$ are much less pronounced in comparison with the model results. This might be the outcome of the discrepancies that was discussed above.

Acknowledgments. The South Pole Ice Crystal Experiment was designed by Paul Lawson (SPEC, Inc. Boulder, Colorado) and was funded in part under 
Grant OPP-9909593 awarded to SPEC Inc. from the U.S. National Science Foundation. This work was also funded by a grant from the CNRS/PATOM committee. We thank P. Zmarzly and D. O'Connor (SPEC Inc.) for their helpful contribution to the SPICE experiment. Thanks are due to J.-F. Fournol (LaMP) for his help in the PN preparation. The authors are grateful to A. Macke for providing his Monte Carlo ray tracing code.

\section{APPENDIX}

\section{Model of the Surface Roughness}

In this section, we follow the notations used in the paper by Yang and Liou (1998). We assume that a rough surface is composed of a number of small facets, which are locally planar and randomly tilted from their positions corresponding to the case of perfectly plane surface. Let the $Z$ axis indicate the direction normal to the mean position of the particle surface. The tilt of the facet is specified by its normal direction that can be given in terms of two angles, $\theta$ and $\varphi$. (Nevertheless the notations are the same; there is nothing common with the scattering angles used in the previous sections.) The slopes of a facet along the $X$ and $Y$ direction are given as follows (Yang and Liou 1998):

$$
\begin{aligned}
& Z_{x}=\frac{\partial Z}{\partial x}=\left(\mu^{-2}-1\right)^{1 / 2} \cos \varphi \\
& Z_{y}=\frac{\partial Z}{\partial y}=\left(\mu^{-2}-1\right)^{1 / 2} \sin \varphi
\end{aligned}
$$

where $\mu=\cos \theta$. The derivatives in Eqs. (A1) and (A2) are confined on the facet.

The tilt distribution is supposed to be azimuthally homogeneous, that is, independent of the angle $\varphi$. We propose to specify the characteristic $\mu$ as a twoparametric one, that is, its probability distribution function should depend on the scale parameter $\sigma$ and the shape parameter $\eta$. The last one determines the kurtosis of the PDF. In a stochastical manner, the roughness characteristics can be specified as follows:

$$
\begin{aligned}
& \mu=1 /\left[1+\sigma^{2}\left(-\ln t_{1}\right)^{1 / \eta}\right]^{1 / 2} \\
& \varphi=2 \pi t_{2}
\end{aligned}
$$

where $t_{1}$ and $t_{2}$ are random numbers distributed uniformly in the range of $(0,1)$.

Considering the relationships (A1)-(A2), the random number generators (A3)-(A4) lead to the following PDF of the slopes of a facet:
$P\left(Z_{x}, Z_{y}\right)=\frac{\eta}{\pi \sigma^{2}}\left(\frac{Z_{x}^{2}+Z_{y}^{2}}{\sigma^{2}}\right)^{\eta-1} \exp \left[-\left(\frac{Z_{x}^{2}+Z_{y}^{2}}{\sigma^{2}}\right)^{\eta}\right]$

It is seen from Eq. (A5) that statistics of the parameter $Z_{x}^{2}+Z_{y}^{2}$ corresponds to the Weibull distribution (Dodson 1994). Accordingly, we consider the proposed model of the surface roughness as based on the Weibull statistics. The Weibull distribution is widely used because of its simplicity and flexibility. It allows not only scaling, but also variation in large range of the shape and kurtosis of the probability distribution function. When the shape parameter $\eta$ takes value of 1.0, Eq. (A5) is reduced to a 2D Gaussian distribution and Eq. (A3) is coincident with Eq. (2.7) of (Yang and Liou 1998).

For other applications that deal with a rough surface, it is useful to present the PDF of $\mu=\cos \theta$

$$
P(\mu)=\frac{2 \eta}{\sigma^{2} \mu^{3}}\left(\frac{\mu^{-2}-1}{\sigma^{2}}\right)^{\eta-1} \exp \left[-\left(\frac{\mu^{-2}-1}{\sigma^{2}}\right)^{\eta}\right],
$$

which corresponds to the random number generator (A3).

\section{REFERENCES}

Auriol, F., J.-F. Gayet, G. Febvre, O. Jourdan, L. Labonnote, and G. Brogniez, 2001: In situ observations of cirrus cloud scattering phase function with $22^{\circ}$ and $46^{\circ}$ halos: Cloud field study on 19 February 1998. J. Atmos. Sci., 58, 3376-3390.

Bacon, N. J., and B. D. Swanson, 2000: Laboratory measurements of light scattering by single levitated ice crystals. J. Atmos. Sci., 57, 2094-2104.

Baran, A. J., 2004: On the scattering and absorption properties of cirrus cloud. J. Quant. Spectrosc. Radiat. Transfer, 89, 17-36.

- V. N. Shcherbakov, B. A. Baker, J.-F. Gayet, and P. R. Lawson, 2005: On the scattering phase function of non-symmetric ice crystals. Quart. J. Roy. Meteor. Soc., 131, 2609-2616.

Barkey, B., M. Bailey, K.-N. Liou, and J. Hallett, 2002: Lightscattering properties of plate and column ice crystals generated in a laboratory cold chamber. Appl. Opt., 41, 5792-5796.

Cox, C., and W. Munk, 1954: Measurement of the roughness of the sea surface from photographs of the sun's glitter. J. Opt. Soc. Amer., 44, 838-850.

Dodson, B., 1994: Weibull Analysis. American Society for Quality Control, $256 \mathrm{pp}$.

Gayet, J.-F., O. Crepel, J.-F. Fournol, and S. Oshchepkov, 1997: A new airborne polar nephelometer for the measurements of optical and microphysical cloud properties. Part I: Theoretical design. Ann. Geophys., 15, 451-459.

_ phase function of stratocumulus, contrails, and cirrus. Geophys. Res. Lett., 25, 971-974.

_, S. Asano, A. Yamazaki, A. Uchiyama, A. Sinyuk, O. Jourdan, and F. Auriol, 2002: Two case studies of continental-type water and maritime mixed-phased stratocumuli over the sea. 
Part I: Microphysical and optical properties. J. Geophys. Res., 107, 4569, doi:10.1029/2001JD001106.

Herbert, G. A., E. R. Green, J. M. Harris, G. L. Koenig, S. J. Roughton, and K. W. Thaut, 1986: Control and monitoring instrumentation for the continuous measurement of atmospheric $\mathrm{CO} 2$ and meteorological variables. J. Atmos. Oceanic Technol., 3, 414-421.

Hesse, E., and Z. Ulanowski, 2003: Scattering from long prisms computed using ray tracing combined with diffraction on facets. J. Quant. Spectrosc. Radiat. Transfer, 79-80, 721-732.

Jourdan, O., S. Oshchepkov, J.-F. Gayet, V. Shcherbakov, and H. Isaka, 2003: Statistical analysis of cloud light scattering and microphysical properties obtained from airborne measurements. J. Geophys. Res., 108, 4155, doi:10.1029/2002JD002723.

Kikuchi, K., and A. W. Hogan, 1979: Properties of diamond dust type ice crystals observed in summer season at AmundsenScott South Pole Station, Antarctica. J. Meteor. Soc. Japan, 57, 180-190.

Kristjánsson, J. E., J. M. Edwards, and D. L. Mitchell, 2000: Impact of a new scheme for optical properties of ice crystals on climates of two GCMs. J. Geophys. Res., 105, $10063-10079$.

Labonnote, L., G. Brogniez, M. Doutriaux-Boucher, J.-C. Buriez, J.-F. Gayet, and H. Chepfer, 2000: Modeling of light scattering in cirrus clouds with inhomogeneous hexagonal monocrystals. Comparison with in-situ and ADEOS-POLDER measurements. Geophys. Res. Lett., 27, 113-116.

Lawson, R. P., 1997: Improved measurements in the mixed clouds and implication to cloud modeling. Proc. WMO Workshop on Measurements of Cloud Properties for Forecast of Weather and Climate, Mexico City, Mexico, WMO, 139-158.

- A. J. Heymsfield, S. M. Aulenbach, and T. L. Jensen, 1998: Shapes, sizes and light scattering properties of ice crystals in cirrus and a persistent contrail during SUCCESS. Geophys. Res. Lett., 25, 1331-1334.

— B. A. Baker, and C. G. Schmitt, 2001: An overview of microphysical properties of Arctic clouds observed in May and July 1998 during FIRE ACE. J. Geophys. Res., 106, 14989 15014.
Liou, K. N., 1986: Influence of cirrus clouds on weather and climate processes: A global perspective. Mon. Wea. Rev, 114, 1167-1199.

- 1992: Radiation and Cloud Processes in the Atmosphere. Oxford University Press, 487 pp.

Macke, A., J. Mueller, and E. Raschke, 1996a: Single scattering properties of atmospheric ice crystals. J. Atmos. Sci., 53, 2813-2825.

_ M. I. Mishchenko, and B. Cairns, 1996b: The influence of inclusions on light scattering by large ice particles. J. Geophys. Res., 101, 23 311-23 316.

Mishchenko, M. I., and A. Macke, 1999: How big should hexagonal ice crystals be to produce halos? Appl. Opt., 38, 16261629.

_, D. J. Wielaard, and B. E. Carlson, 1997: T-matrix computations of zenith-enhanced lidar backscatter from horizontally oriented ice plates. Geophys. Res. Lett., 24, 771-774.

— J. W. Hovenier, and L. D. Travis, Eds., 2000: Light Scattering by Nonspherical Particles: Theory, Measurements, and Applications. Academic Press, 690 pp.

Pluchino, A., 1986: Observations of halo scattering from single ice crystals. Opt. Lett., 11, 276-278.

Rao, R. M., and A. S. Bopardikar, 1998: Wavelet Transforms: Introduction to Theory \& Applications. Book and disk ed. Prentice Hall, 336 pp.

Stephens, G. L., S.-C. Tsay, P. W. Stackhouse Jr., and P. J. Flatau, 1990: The relevance of the microphysical and radiative properties of cirrus clouds to climate and climatic feedback. $J$. Atmos. Sci., 47, 1742-1754.

Ulanowski, Z., E. Hesse, P. H. Kaye, A. J. Baran, and R. Chandrasekhar, 2003: Scattering of light from atmospheric ice analogues. J. Quant. Spectrosc. Radiat. Transfer, 79-80C, 10911102.

Warren, S. G., 1984: Optical constants of ice from the ultraviolet to the microwave. Appl. Opt., 23, 1206-1225.

Yang, P., and K. N. Liou, 1998: Single-scattering properties of complex ice crystals in terrestrial atmosphere. Contr. Atmos. Phys., 71, 223-248. 